\title{
THE KIDNEY AS A SOURCE OF BLOOD AMMONIA IN PATIENTS WITH LIVER DISEASE: THE EFFECT OF ACETAZOLAMIDE * $\dagger$
}

\author{
By EDWARD E. OWEN, MALCOLM P. TYOR, JOHN F. FLANAGAN AND \\ J. NORMAN BERRY \\ (From the Departments of Medicine, Duke University Medical Center and the Veterans \\ Administration Hospital, Durham, N. C.)
}

(Submitted for publication July 14, 1959; accepted October 8, 1959)

The role of the gastrointestinal tract as a major source of blood ammonia has been extensively studied in animals and in patients with liver disease. Less information is available concerning the relative contribution to the blood ammonia by other organs, such as muscle, liver, brain and kidney, which are known to be concerned with ammonia metabolism (1). The renal release of ammonia into the systemic circulation was first demonstrated by the observations of Nash and Benedict in 1921 (2). Although the magnitude of this ammonia release and its effect on the arterial ammonia concentration have not been determined, factors affecting the quantity of ammonia excreted into urine have engendered considerable investigation. Current evidence indicates that changes in urine $\mathrm{pH}$ are important determinants of urinary ammonia excretion, lesser amounts of ammonia appearing in alkaline than in acid urines (3-8). Studies in unilaterally nephrectomized dogs suggest that total renal ammonia production may remain unchanged following acute alterations in urine $\mathrm{pH}$ (9). Of particular interest in this regard have been the observations of significant increases in arterial ammonia concentrations of cirrhotic patients given Diamox (acetazolamide) $(10-12)$, an agent which is known to increase urine $\mathrm{pH}$.

The present investigation is concerned with the release of ammonia into the renal vein of patients with liver disease, with particular emphasis on the effect of intravenous acetazolamide.

\section{METHODS}

Eleven patients with liver disease were studied. All were hospitalized males, ranging in age from 32 to 63

* This investigation was supported (in part) by a research grant (A-3255) from the National Institutes of Health, Bethesda, Md.

† This paper was presented (in part) at the National Meeting of the American Federation for Clinical Research, Atlantic City, N. J., May 3, 1959. years. Nine had Laennec's cirrhosis confirmed by biopsy and two had fatty metamorphosis. Three of the cirrhotic patients (W.F., W.N. and W.S.) exhibited precoma at the time of the study; none showed evidence of fluid retention or of progressive hepatic deterioration. Renal function was normal in all patients.

Patients were studied in the recumbent position after an overnight fast. A constant intravenous infusion (Bowman pump), which delivered 14 to $16 \mathrm{mg}$ of para-amino hippurate $(\mathrm{PAH})$, was maintained throughout the study and was preceded by a priming dose of $\mathrm{PAH}$ calculated to provide plasma levels of $2 \mathrm{mg}$ per $100 \mathrm{ml}$. Arterial samples were obtained from a brachial artery, and renal venous samples from the right renal vein by way of an intravenous no. 8 catheter. Renal venous blood was initially identified from catheter position and apparent arterialization; subsequent confirmation was obtained from oxygen saturations and $\mathrm{PAH}$ extraction. Resting arterial and renal venous samples were obtained 45 to 60 minutes after the $\mathrm{PAH}$ infusion was begun and again at 10,20,30,45, and 60 minutes after the intravenous administration of $500 \mathrm{mg}$ of acetazolamide. Five subjects ingested $1 \mathrm{~L}$ of water 30 to 45 minutes prior to the start of the study. A water diuresis was subsequently maintained by the intravenous infusion of 5 per cent dextrose and water administered at a rate of 20 to $30 \mathrm{ml}$ per minute. In these patients, urine samples were collected anaerobically from an indwelling Foley catheter over time intervals which corresponded to the intervals between blood samples and included two 10-minute control periods. Urine was collected under an oil-toluene mixture. Blood sampling times usually occupied 30 to 45 seconds and were begun at the approximate midpoint of the urine collection period; urine collection generally required 30 to 60 seconds.

Blood ammonia $\left(\mathrm{NH}_{3}\right)$ was measured by a modification of the microdiffusion method of Brown and associates (13) and of Tyor and Wilson (14). Urine ammonia $\left(\mathrm{NH}_{3}\right)$ was measured in duplicate, within 24 hours after sampling, by a similar modification of the same method, using $1 \mathrm{ml}$ aliquots of urine which were appropriately diluted (1:25 or $1: 50$ in control specimens, $1: 10$ or $1: 25$ in post-acetazolamide samples). PAH concentrations in the blood, urine and infusion media were determined by the method of Selkurt (15) with the $\mathrm{N}$-naphthyl ethylenediamine dihydrochloride recrystallized with hydrochloric acid, as described by Bratton and Marshall (16). The $\mathrm{pH}$ of whole blood and urine were measured with a Cambridge Model $\mathrm{R} \mathrm{pH}$ meter equipped 
with an enclosed glass electrode. Measurements were made at room temperature, usually 25 to $26^{\circ} \mathrm{C}$, and the results corrected to $37^{\circ}$ by Rosenthal's factor (17). The carbon dioxide content of whole blood was determined by the method of Van Slyke and Neill (18). The carbon dioxide tension was calculated from this value, the $\mathrm{pH}$ and the hemoglobin concentration by the line chart of Van Slyke and Sendroy (19), using a pK of 6.11. Oxygen content and saturation were determined by the spectrophotometric method of Hickam and Frayser (20). Hematocrits were determined in duplicate from arterial and renal venous blood in Wintrobe tubes centrifuged at 3,000 rpm for 30 minutes.

Renal plasma flow (RPF) was calculated by a modification of the Fick principle, using the formula 1) RPF = IR/A-R, where IR represents the infusion rate of $\mathrm{PAH}$ in milligrams per minute, $A$ and $R$ represent the $P A H$ concentration in arterial and renal-venous blood in milligrams per milliliter. This principle and formula have been previously described and utilized by Bradley, Ingelfinger, Bradley and Curry (21) in the determination of hepatic plasma flow, using sulfobromophthalein. Since the arterial PAH concentrations remained essentially constant throughout the entire period of observation, the $\mathrm{PAH}$ removal by the kidneys may be considered equal to the rate of $\mathrm{PAH}$ infusion. The validity of this method is also dependent upon the assumption that the PAH level in a sample of blood taken from the right renal vein is representative of the level in the left renal vein. Five of the patients had simultaneous measurements of $R P F$ by standard clearance procedure 2), $C^{\mathbf{P A B}}=U \mathrm{UV} / \mathrm{P}$. In these subjects, RPF was also calculated from the formula 3), RPF = UV/A-R, as described by Cargill (22), where UV is the rate of urinary excretion of PAH in milligrams per minute. In each instance, RPF was converted to renal blood flow (milliliters per minute), which was calculated from the formula $R B F=R P F /$ (1-Hct.). Values obtained using Formula 1 averaged $1,919 \mathrm{ml}$ per minute. Similar average values were obtained when RBF was calculated from Formula 3, $1,802 \mathrm{ml}$ per minute. However, calculation of $\mathrm{RBF}$ by standard clearance methods, Formula 2, yielded lower average values, $1,362 \mathrm{ml}$ per minute. $\mathrm{PAH}$ extraction in the 5 patients who had simultaneous measurements averaged 85 per cent. The extraction of $\mathrm{PAH}\left(\mathrm{E}^{\mathrm{PAH}}\right)$ was calculated from the formula $\mathrm{E}^{\mathrm{PAB}}=$ $(\mathrm{A}-\mathrm{R} / \mathrm{A}) \times 100$, where $\mathrm{A}$ is arterial $\mathrm{PAH}$ concentration in milligrams per milliliter and $R$ is renal venous PAH concentration in milligrams per milliliter. Values for $E^{P A B}$ in the 11 patients studied ranged from 75 to 92 per cent, mean $=87$ per cent. Release of ammonia into the renal vein (micrograms per minute) was calculated from the RBF and arteriovenous renal ammonia difference in micrograms per milliliter; the ammonia excretion into the urine (micrograms per minute) from urine volume in milliliters per minute; and the ammonia concentration in micrograms per milliliter. The RBF and arteriovenous renal oxygen differences in volumes per cent were used to calculate renal oxygen consumption (milliliters per minute).

\section{RESULTS}

The data obtained from each patient as well as mean values before and after acetazolamide administration are presented in Tables I and II. The mean arterial ammonia concentration increased significantly 20 minutes after intravenous acetazolamide and attained a maximum increase over resting values, $30 \mu \mathrm{g}$ per $100 \mathrm{ml}$, at 45 minutes. Patient W. N., who exhibited marked resting hyperammonemia and a maximum increase in arterial ammonia concentration of $60 \mu \mathrm{g}$ per $100 \mathrm{ml}$, was the only subject who showed overt cerebral deterioration during the study. Frank coma responded readily to routine measures.

Resting renal arteriovenous ammonia differences were negative in 10 of 11 subjects, indicating release of ammonia into the renal vein. A twofold increase in mean renal ammonia release $(p=<$ 0.01 ) was exhibited within ten minutes after acetazolamide administration and was maintained throughout the remainder of the study. Renal blood flow was essentially unchanged throughout the entire period of observation. The abrupt increase in the release of ammonia into the renal vein was associated with an equally rapid decrease in urine ammonia excretion in the five patients who had simultaneous measurements. In these subjects, mean resting urine ammonia excretion decreased significantly from $1,590 \mu \mathrm{g}$ per minute to $1,096 \mu \mathrm{g}$ per minute at the 10 minute period and to $360 \mu \mathrm{g}$ per minute 20 minutes after acetazolamide, remaining essentially unchanged thereafter. Although maximum reduction of urine ammonia excretion was observed at 20 minutes, an earlier effect would seem likely, since the 10 minute urine value reflected the tubular ammonia concentration immediately before and after acetazolamide. This reduction in urine ammonia excretion could be readily correlated with a rise in urine $\mathrm{pH}$. Mean urine $\mathrm{pH}$ attained maximum alkalinity at $20 \mathrm{~min}$ utes and was essentially unchanged throughout the remainder of the study. A significant decrease in mean arterial $\mathrm{pH}$ was observed at the 45 minute period.

Renal oxygen consumption increased significantly $(\mathrm{p}=<0.01)$ at 20 minutes but was similar to the resting value at 45 minutes. No explanation for this observation is apparent at this time. 
TABLE I

The effect of intravenous acetazolamide on the release of ammonia by the kidney

\begin{tabular}{|c|c|c|c|c|c|c|c|c|c|c|c|c|c|}
\hline \multirow[b]{2}{*}{ Patient } & \multirow[b]{2}{*}{ Time } & \multirow[b]{2}{*}{$\begin{array}{c}\text { Arterial } \\
\mathrm{NH}_{3}\end{array}$} & \multirow{2}{*}{$\begin{array}{c}\text { Renal } \\
\mathrm{NH}_{3} \\
\mathrm{~A}-\mathrm{V}\end{array}$} & \multirow{2}{*}{$\begin{array}{c}\text { Renal } \\
\mathrm{O}_{2} \\
\mathrm{~A}-\mathrm{V}\end{array}$} & \multirow{2}{*}{$\begin{array}{c}\text { Renal } \\
\text { blood } \\
\text { flow }\end{array}$} & \multirow{2}{*}{$\begin{array}{c}\text { Release } \\
\text { of } \mathrm{NH}_{3} \\
\text { into } \\
\text { renal vein }\end{array}$} & \multirow{2}{*}{$\begin{array}{l}\text { Urine } \\
\mathrm{NH}_{3} \\
\text { excre- } \\
\text { tion }\end{array}$} & \multirow{2}{*}{$\begin{array}{l}\text { Total } \\
\text { renal } \\
\mathrm{NH}_{3} \\
\text { release }\end{array}$} & \multirow{2}{*}{$\begin{array}{c}\text { Renal } \\
\mathrm{O}_{2} \\
\text { consump- } \\
\text { tion }\end{array}$} & \multirow[b]{2}{*}{$\begin{array}{c}\text { Urine } \\
\text { pH }\end{array}$} & \multicolumn{3}{|c|}{ Arterial } \\
\hline & & & & & & & & & & & $\mathrm{pH}$ & $\begin{array}{c}\text { Serum } \\
\mathrm{CO}_{2}\end{array}$ & $\mathrm{pCO}_{2}$ \\
\hline & $\min$ & $\mu g / 100 \mathrm{ml}$ & $\mu \mathrm{g} / 100 \mathrm{ml}$ & vol \% & $m l / m i n$ & $\mu g / \min \dagger$ & $\mu g / m i n_{+}^{+}$ & $\mu g / m i n$ & $m l / m i n$ & & & $20 \mathrm{Cl}$ & $\mathrm{mm} \mathrm{Hg}$ \\
\hline & $\underset{*}{\text { Rest }}$ & 168 & -43 & 1.17 & 1,555 & 1,337 & 1,248 & 2,585 & 18.2 & 6.25 & 7.37 & 49.1 & 40.8 \\
\hline \multirow[t]{3}{*}{ W. s. } & $\begin{array}{l}10 \\
20 \\
30\end{array}$ & $\begin{array}{l}215 \\
212 \\
223\end{array}$ & $\begin{array}{l}-111 \\
-88 \\
-81\end{array}$ & 1.00 & $\begin{array}{l}1,709 \\
1,752 \\
1,947\end{array}$ & $\begin{array}{l}3,794 \\
3,084 \\
3,154\end{array}$ & $\begin{array}{l}274 \\
189 \\
196\end{array}$ & $\begin{array}{l}4,068 \\
3,273 \\
3,350\end{array}$ & 17.5 & $\begin{array}{l}7.38 \\
7.31 \\
7.29\end{array}$ & 7.33 & 45.8 & 38.4 \\
\hline & $\begin{array}{l}45 \\
60\end{array}$ & $\begin{array}{l}230 \\
255\end{array}$ & $\begin{array}{r}01 \\
-73 \\
-102\end{array}$ & 0.72 & $\begin{array}{l}2,122 \\
2,120\end{array}$ & $\begin{array}{l}3,098 \\
4,325\end{array}$ & $\begin{array}{l}173 \\
272\end{array}$ & $\begin{array}{l}3,271 \\
4,597\end{array}$ & 16.4 & $\begin{array}{l}7.23 \\
7.22\end{array}$ & 7.34 & 44.1 & 36.5 \\
\hline & $\underset{*}{\text { Rest }}$ & 114 & -12 & 1.21 & 2,000 & 480 & 3,178 & 3,658 & 24.0 & 4.99 & 7.45 & 49.0 & 32.0 \\
\hline \multirow[t]{3}{*}{ (i. J. } & $\begin{array}{l}10 \\
20 \\
30\end{array}$ & $\begin{array}{l}135 \\
140 \\
147\end{array}$ & $\begin{array}{l}-64 \\
=60 \\
-\quad 56\end{array}$ & 1.58 & $\begin{array}{l}2,034 \\
2,044 \\
2,034\end{array}$ & $\begin{array}{l}2,604 \\
2,453 \\
2,277\end{array}$ & $\begin{array}{r}2,715 \\
710 \\
300\end{array}$ & $\begin{array}{l}5,319 \\
3,163 \\
2,577\end{array}$ & 32.1 & $\begin{array}{l}6.83 \\
7.35 \\
7.34\end{array}$ & 7.45 & 46.8 & 30.3 \\
\hline & $\begin{array}{l}45 \\
60\end{array}$ & $\begin{array}{l}146 \\
144\end{array}$ & $\begin{array}{l}-61 \\
-67\end{array}$ & $\begin{array}{l}1.38 \\
1.44\end{array}$ & $\begin{array}{l}1,868 \\
1,881\end{array}$ & $\begin{array}{l}2,279 \\
2,521\end{array}$ & $\begin{array}{l}288 \\
209\end{array}$ & $\begin{array}{l}2,567 \\
2,830\end{array}$ & $\begin{array}{l}25.7 \\
27.0\end{array}$ & $\begin{array}{l}7.30 \\
7.43\end{array}$ & $\begin{array}{l}7.44 \\
7.43\end{array}$ & $\begin{array}{l}45.7 \\
45.0\end{array}$ & $\begin{array}{l}30.2 \\
30.6\end{array}$ \\
\hline & $\underset{*}{\text { Rest }}$ & 78 & -73 & 0.60 & 1,432 & 2,090 & 1,880 & 3,930 & 8.6 & 6.65 & 7.42 & 52.5 & 36.5 \\
\hline \multirow[t]{3}{*}{ W. M. } & $\begin{array}{l}10 \\
20 \\
30\end{array}$ & $\begin{array}{r}89 \\
96 \\
117\end{array}$ & $\begin{array}{l}-83 \\
-108 \\
-96\end{array}$ & 0.96 & 1,525 & 3,294 & $\begin{array}{r}1,192 \\
453 \\
202\end{array}$ & 3,727 & 14.6 & $\begin{array}{l}7.33 \\
7.42 \\
7.40\end{array}$ & 7.38 & 51.1 & 39.1 \\
\hline & $\begin{array}{l}45 \\
60\end{array}$ & $\begin{array}{l}105 \\
116\end{array}$ & $\begin{array}{l}-95 \\
-92\end{array}$ & $\begin{array}{l}0.88 \\
0.88\end{array}$ & $\begin{array}{r}993 \\
1,040\end{array}$ & $\begin{array}{l}1,887 \\
1,907\end{array}$ & $\begin{array}{l}188 \\
126\end{array}$ & $\begin{array}{l}2,009 \\
2,033\end{array}$ & 8.7 & $\begin{array}{l}7.40 \\
7.39\end{array}$ & $\begin{array}{l}7.38 \\
7.38\end{array}$ & $\begin{array}{l}50.0 \\
49.0\end{array}$ & $\begin{array}{l}38.0 \\
36.9\end{array}$ \\
\hline & $\underset{*}{\text { Rest }}$ & 95 & -9 & 1.05 & 1,222 & 219 & 1,184 & 1,403 & 12.8 & 7.15 & 7.35 & 49.2 & 40.0 \\
\hline \multirow[t]{4}{*}{$\mathrm{J} . \mathrm{P}$} & $\begin{array}{l}10 \\
20\end{array}$ & 112 & -11 & 1.09 & $\begin{array}{l}1,312 \\
1,436\end{array}$ & 316 & & 1,223 & 15.7 & & 7.34 & 47.1 & 39.4 \\
\hline & $\begin{array}{l}30 \\
45\end{array}$ & $\begin{array}{l}103 \\
120\end{array}$ & $\begin{array}{l}-31 \\
-\quad 5\end{array}$ & & $\begin{array}{l}1,462 \\
1,429\end{array}$ & $\begin{array}{l}906 \\
143\end{array}$ & $\begin{array}{r}843 \\
1,008\end{array}$ & 1,749 & & $\begin{array}{l}7.30 \\
7.28\end{array}$ & $2 \pi$ & 480 & 397 \\
\hline & 60 & 124 & -26 & & 1,482 & 771 & & & & & 1.21 & 40.0 & 59.1 \\
\hline & $\underset{*}{\text { Rest }}$ & 72 & -35 & 1.11 & 1,639 & 1,147 & 511 & 1,658 & 18.2 & 6.39 & 7.3 .3 & 48.7 & 41.2 \\
\hline \multirow[t]{2}{*}{ A. H. } & $\begin{array}{l}10 \\
20 \\
30 \\
45\end{array}$ & $\begin{array}{l}72 \\
92 \\
92 \\
82\end{array}$ & $\begin{array}{l}-60 \\
=40 \\
-\quad 37 \\
-\quad 47\end{array}$ & $\begin{array}{l}1.19 \\
0.28\end{array}$ & $\begin{array}{l}1,577 \\
1,766 \\
1,955 \\
1,849\end{array}$ & $\begin{array}{l}1,892 \\
1,421 \\
1,447 \\
1,738\end{array}$ & $\begin{array}{r}335 \\
96 \\
81 \\
52\end{array}$ & $\begin{array}{l}2,227 \\
1,517 \\
1,528 \\
1,790\end{array}$ & $\begin{array}{r}21.0 \\
5.1\end{array}$ & $\begin{array}{l}7.39 \\
7.40 \\
7.40 \\
7.34\end{array}$ & $\begin{array}{l}7.34 \\
7.35\end{array}$ & $\begin{array}{l}46.7 \\
46.6\end{array}$ & $\begin{array}{l}39.0 \\
38.5\end{array}$ \\
\hline & $\underset{*}{\operatorname{Rest}}$ & 277 & & 0.72 & 1,618 & & & & 11.6 & 5.50 & 7.34 & 38.1 & 32.4 \\
\hline \multirow[t]{3}{*}{ W.N. } & $\begin{array}{l}10 \\
20 \\
30\end{array}$ & $\begin{array}{l}291 \\
304 \\
311\end{array}$ & $\begin{array}{l}-16 \\
=42 \\
-41\end{array}$ & 0.90 & $\begin{array}{l}1,579 \\
1,579 \\
1,654\end{array}$ & $\begin{array}{r}505 \\
1.326 \\
1.356\end{array}$ & & & 14.2 & & 7.31 & 33.7 & 29.6 \\
\hline & $\begin{array}{l}30 \\
45\end{array}$ & $\begin{array}{l}311 \\
337\end{array}$ & $\begin{array}{l}-41 \\
-34\end{array}$ & 0.72 & $\begin{array}{l}1,654 \\
1,787\end{array}$ & $\begin{array}{l}1,356 \\
1,215\end{array}$ & & & 12.9 & & 7.30 & 31.7 & 27.9 \\
\hline & $\underset{*}{\text { Rest }}$ & 93 & -33 & 1.21 & 983 & 649 & & & 11.9 & & 7.33 & 53.0 & 44.0 \\
\hline \multirow[t]{4}{*}{ J. W: } & $\begin{array}{l}10 \\
20 \\
30\end{array}$ & $\begin{array}{r}108 \\
86 \\
94\end{array}$ & $\begin{array}{l}-65 \\
-106 \\
-\quad 99\end{array}$ & 1.58 & $\begin{array}{r}1,013 \\
968 \\
870\end{array}$ & $\begin{array}{l}1,316 \\
2,052 \\
1,722\end{array}$ & & & 15.3 & & 7.34 & 54.2 & 43.0 \\
\hline & $\begin{array}{l}45 \\
60\end{array}$ & $\begin{array}{l}106 \\
104\end{array}$ & $\begin{array}{l}-111 \\
-106\end{array}$ & $\begin{array}{l}1.38 \\
1.44\end{array}$ & $\begin{array}{l}897 \\
846\end{array}$ & $\begin{array}{l}1,991 \\
1,793\end{array}$ & & & 12.4 & & 7.32 & 52.8 & 46.1 \\
\hline & & & & & & & & & 12.2 & & 7.33 & 51.5 & 43.9 \\
\hline & $\underset{*}{\text { Rest }}$ & 83 & -64 & 0.86 & 932 & 1,193 & & & 8.0 & & 7.40 & 49.4 & 36.1 \\
\hline \multirow[t]{2}{*}{ J. B. } & $\begin{array}{l}10 \\
20 \\
30\end{array}$ & $\begin{array}{l}90 \\
86\end{array}$ & $\begin{array}{l}-99 \\
-86\end{array}$ & $\begin{array}{l}0.72 \\
1.86\end{array}$ & $\begin{array}{l}1,047 \\
1,109\end{array}$ & $\begin{array}{l}2,073 \\
1,907\end{array}$ & & & $\begin{array}{r}7.5 \\
20.5\end{array}$ & & $\begin{array}{l}7.33 \\
7.36\end{array}$ & 50.1 & 39.4 \\
\hline & $\underset{*}{\text { Rest }}$ & 135 & -50 & 0.92 & 1,447 & 1,447 & & & 13.3 & 7.0 & 7.38 & 53.0 & 45.7 \\
\hline \multirow[t]{3}{*}{ W. W. } & $\begin{array}{l}10 \\
20 \\
30\end{array}$ & $\begin{array}{l}121 \\
121 \\
109\end{array}$ & $\begin{array}{l}-59 \\
-\quad 34 \\
-\quad 61\end{array}$ & 1.07 & $\begin{array}{l}1,279 \\
1,536 \\
1,440\end{array}$ & $\begin{array}{l}1,505 \\
1,044 \\
1,757\end{array}$ & & & 16.4 & & 7.32 & 54.2 & 47.3 \\
\hline & $\begin{array}{l}45 \\
60\end{array}$ & $\begin{array}{l}129 \\
146\end{array}$ & $\begin{array}{l}-64 \\
-67\end{array}$ & $\begin{array}{l}1.13 \\
0.88\end{array}$ & $\begin{array}{l}1,511 \\
1,477\end{array}$ & $\begin{array}{l}1,934 \\
1,979\end{array}$ & & & 17.1 & & $\begin{array}{l}7.31 \\
7.32\end{array}$ & $\begin{array}{l}52.8 \\
51.5\end{array}$ & $\begin{array}{l}46.9 \\
44.2\end{array}$ \\
\hline & $\underset{*}{\operatorname{Rest}}$ & 203 & -2 & 1.46 & 1,684 & 67 & & & 24.5 & 6.30 & 7.45 & 55.4 & 36.1 \\
\hline \multirow[t]{3}{*}{ W. F. } & $\begin{array}{l}10 \\
20 \\
30\end{array}$ & $\begin{array}{l}214 \\
206 \\
233\end{array}$ & $\begin{array}{l}-38 \\
=51 \\
-51\end{array}$ & 1.87 & $\begin{array}{l}1,501 \\
1,466 \\
1,461\end{array}$ & $\begin{array}{l}1,441 \\
1,495 \\
1,665\end{array}$ & & & 27.4 & & 7.46 & 54.2 & 34.6 \\
\hline & $\begin{array}{l}45 \\
60\end{array}$ & $\begin{array}{l}224 \\
226\end{array}$ & $\begin{array}{l}-5.3 \\
-44\end{array}$ & 1.62 & $\begin{array}{l}1,841 \\
2,311\end{array}$ & $\begin{array}{l}1,951 \\
2,034\end{array}$ & & & 29.8 & & $\begin{array}{l}7.42 \\
7.43\end{array}$ & $\begin{array}{l}52.7 \\
51.7\end{array}$ & $\begin{array}{l}36.8 \\
35.4\end{array}$ \\
\hline & $\underset{*}{\operatorname{Rest}}$ & 101 & -33 & 1.26 & 1,191 & 786 & & & & & & & \\
\hline \multirow[t]{3}{*}{ R. M. } & $\begin{array}{l}10 \\
20\end{array}$ & $\begin{array}{l}100 \\
113\end{array}$ & $\begin{array}{l}-52 \\
-64\end{array}$ & 1.61 & $\begin{array}{l}1,162 \\
1,088\end{array}$ & $\begin{array}{l}1,208 \\
1,395\end{array}$ & & & 15.0 & & 7.39 & 53.0 & 39.5 \\
\hline & 30 & 113 & -67 & & 1,385 & 1,856 & & & 17.5 & & 7.38 & 53.0 & 40.0 \\
\hline & $\begin{array}{l}45 \\
60\end{array}$ & $\begin{array}{l}120 \\
124\end{array}$ & $\begin{array}{l}-53 \\
-51\end{array}$ & 1.38 & $\begin{array}{l}1,190 \\
1,103\end{array}$ & $\begin{array}{l}1,155 \\
1,023\end{array}$ & & & 16.4 & & 7.41 & 49.1 & 35.0 \\
\hline
\end{tabular}

* Diamox (acetazolamide) $500 \mathrm{mg}$ iv.

† Instantaneous values obtained at indicated time periods.

t Instantaneous values obtained at indicated time periods.
+ Average excretion per minute collected throughout indicated time periods. 
TABLE II

Comparison of mean values for renal ammonia release after intravenous acetazolamide

\begin{tabular}{|c|c|c|c|c|c|c|c|c|c|c|c|c|}
\hline \multirow[b]{2}{*}{ Time } & & \multirow[b]{2}{*}{$\underset{\mathrm{NH}_{3}}{\text { Arterial }}$} & \multirow{2}{*}{$\begin{array}{l}\text { Renal } \\
\text { NHa } \\
\mathbf{A}-\mathbf{V}\end{array}$} & \multirow{2}{*}{$\begin{array}{c}\text { Renal } \\
\mathrm{O}_{\mathbf{2}} \\
\mathbf{A}-\mathbf{V}\end{array}$} & \multirow{2}{*}{$\begin{array}{l}\text { Renal } \\
\text { blood } \\
\text { flow }\end{array}$} & \multirow{2}{*}{$\begin{array}{l}\text { Release } \\
\text { of NH: } \\
\text { into } \\
\text { renal vein }\end{array}$} & \multirow{2}{*}{$\begin{array}{c}\text { Urine } \\
\text { NHs } \\
\text { excre- } \\
\text { tion }\end{array}$} & \multirow{2}{*}{ 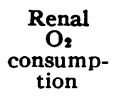 } & \multirow[b]{2}{*}{$\underset{\mathrm{pH}}{\text { Urine }}$} & \multicolumn{3}{|c|}{ Arterial } \\
\hline & & & & & & & & & & $\mathrm{pH}$ & $\underset{\mathrm{CO}_{2}}{\text { Serum }}$ & $\mathrm{pCO}_{2}$ \\
\hline $\begin{array}{l}\min \\
\text { Rest }\end{array}$ & $\overline{\mathrm{d}} \boldsymbol{t}$ & $\begin{array}{c}\mu g / 100 \mathrm{ml} \\
129\end{array}$ & $\begin{array}{c}\mu g / 100 m l \\
-32.2\end{array}$ & $\begin{array}{r}\text { vol \% } \\
1.05\end{array}$ & $\begin{array}{c}m l / m i n \\
1,430\end{array}$ & $\begin{array}{r}\mu g / \min \\
856\end{array}$ & $\begin{array}{r}\mu g / \min \\
1,590\end{array}$ & $\begin{array}{c}\mathrm{ml} / \mathrm{min} \\
15.1\end{array}$ & 6.29 & 7.38 & $\begin{array}{r}\text { vol \% } \\
50.5\end{array}$ & $\begin{array}{c}m m H_{g} \\
38.6\end{array}$ \\
\hline $10^{*}$ & $\begin{array}{l}\bar{d} \\
\mathrm{p} \ddagger\end{array}$ & $\begin{array}{l}149 \\
<0.1\end{array}$ & $\begin{array}{l}-60.9 \\
<0.01\end{array}$ & & $\begin{array}{r}1,462 \\
>0.1\end{array}$ & $\begin{array}{r}1,784 \\
<0.01\end{array}$ & $\begin{array}{r}1,096 \\
<0.05\end{array}$ & & $\begin{array}{r}7.21 \\
<0.05\end{array}$ & & & \\
\hline $20 *$ & $\begin{array}{l}\bar{d} \\
\mathbf{p}\end{array}$ & $\begin{array}{r}143 \\
<0.02\end{array}$ & $\begin{array}{l}-63.9 \\
<0.01\end{array}$ & $\begin{array}{r}1.23 \\
<0.01\end{array}$ & $\begin{array}{l}1,466 \\
>0.1\end{array}$ & $\begin{array}{r}1,814 \\
<0.01\end{array}$ & 362 & $\begin{array}{l}24.3 \\
<0.01\end{array}$ & 7.37 & $\begin{array}{l}7.36 \\
>0.1\end{array}$ & $\begin{array}{l}49.1 \\
<0.01\end{array}$ & $\begin{array}{r}38.1 \\
>0.1\end{array}$ \\
\hline $30^{*}$ & $\begin{array}{l}\bar{d} \\
\mathbf{p}\end{array}$ & $\begin{array}{r}148 \\
<0.02\end{array}$ & $\begin{array}{l}-64.2 \\
<0.01\end{array}$ & & $\begin{array}{r}1,549 \\
>0.1\end{array}$ & $\begin{array}{r}1,805 \\
<0.01\end{array}$ & $\begin{array}{r}324 \\
<0.05\end{array}$ & & $\begin{array}{r}7.35 \\
<0.05\end{array}$ & & & \\
\hline $45 *$ & $\begin{array}{l}\bar{d} \\
\mathbf{p}\end{array}$ & $\begin{array}{r}160 \\
<0.01\end{array}$ & $\begin{array}{l}-59.6 \\
<0.01\end{array}$ & $>0.1$ & $\begin{array}{r}1,549 \\
>0.1\end{array}$ & $\begin{array}{l}1,742 \\
<0.01\end{array}$ & $\begin{array}{r}342 \\
<0.1\end{array}$ & $\stackrel{16}{>0.1}$ & $\begin{array}{r}7.31 \\
<0.02\end{array}$ & $\begin{array}{r}7.35 \\
<0.02\end{array}$ & $\begin{array}{l}47.5 \\
<0.01\end{array}$ & $\begin{array}{r}37.6 \\
>0.1\end{array}$ \\
\hline $60^{*}$ & $\begin{array}{l}\bar{d} \\
p\end{array}$ & $\begin{array}{r}155 \\
<0.01\end{array}$ & $\begin{array}{c}-69.4 \\
<0.01\end{array}$ & & $\begin{array}{r}1,533 \\
>0.1\end{array}$ & $\begin{array}{r}2,045 \\
<0.01\end{array}$ & 202 & & 7.35 & & & \\
\hline
\end{tabular}

* Minutes after intravenous acetazolamide.

Mean values at the time periods indicated.
$\ddagger$ Significance as compared with resting values.

\section{DISCUSSION}

The present study demonstrates that the kidney normally releases a variable amount of ammonia into the systemic circulation of patients with liver disease. In addition, it is apparent from these data that the intravenous administration of acetazolamide to such patients results in a prompt and sustained increase of this renal contribution, which is associated with a rise in arterial ammonia concentration. Previous comparisons of peripheral venous and arterial ammonia values with renal venous levels in dogs $(2,23,24)$ and in humans $(25,26)$ have shown in almost every instance a greater ammonia concentration in renal venous blood. The present observations emphasize the magnitude of this renal contribution in resting patients with liver disease. Indeed, serial determinations of arteriovenous ammonia differences across the periphery, liver and kidney in resting normal subjects and in patients with liver disease without hyperammonemia suggest that the kidney may be a major source of the "normal" blood ammonia (27).

Patients with liver disease whose gastrointestinal ammonia production appears to be negligible, may exhibit progressive increases in arterial ammonia concentration $(28,29)$. Similar elevations have been observed in such patients following oral and intravenous acetazolamide $(10-12)$. In these instances, the increased increment of arterial ammonia has been attributed either to a greater endo- genous ammonia production or to a decrease in organ removal. Muscle, liver, brain and kidney are potential ammonia donors and receivers (1). However, with the exception of the kidney, arteriovenous ammonia differences across these sites have been primarily positive in patients with liver disease and hyperammonemia, suggesting that these organs function chiefly as ammonia receivers (2531). Dawson, de Groote, Rosenthal and Sherlock have reported increased arterial ammonia concentrations and unchanged arteriorenal venous ammonia differences in three cirrhotic patients two hours after the oral ingestion of acetazolamide (11). In contrast, this report suggests that the kidney may be a source of the observed increased increment of arterial ammonia which followed the intravenous administration of acetazolamide to patients with liver disease with or without pre-existing hyperammonemia. The relationship of this increased renal ammonia contribution to the observed elevation of arterial ammonia concentration becomes more apparent from the following theoretical considerations. If one assumes an average extracellular fluid volume of $14 \mathrm{~L}$ and unchanged organ ammonia removal, the increased increment of ammonia released into the renal vein, which averaged $982 \mu \mathrm{g}$ per minute for the 60 minute period after acetazolamide, could account for as much as a $420 \mu \mathrm{g}$ per $100 \mathrm{ml}$ increase in extracellular fluid ammonia concentration. Actually, the observed average increase in arterial am- 
monia concentration at 60 minutes was $26 \mu \mathrm{g}$ per $100 \mathrm{ml}$. That this increased increment of ammonia released into the renal vein may elevate the arterial ammonia concentration in patients with liver disease is apparent from the results obtained in four cirrhotic patients given $1 \mathrm{mg}$ per minute of ammonia as ammonium chloride intravenously; arterial ammonia concentration increased rapidly in each subject, with an average increase of 180 $\mu \mathrm{g}$ per $100 \mathrm{ml}$ at the 60 minute period (32).

The arterial ammonia concentration at any instant is the resultant of a number of factors, including ammonia production by tissues, hepatic removal, and rate of diffusion into and out of cells. There is no reason to assume that acetazolamide affects only the first-named parameter. Indeed, a decreased removal of ammonia by the periphery and unchanged hepatic uptake have been reported in cirrhotics following oral and intravenous acetazolamide (11). An increased cerebral removal has also been noted (12). Nevertheless, the present studies indicate that the increased renal output of ammonia following acetazolamide is more than sufficient to provide the extra ammonia to serve as an ultimate source for the blood increment.

The prompt twofold increase in the release of ammonia into the renal vein, which followed acetazolamide administration, was associated with an equally rapid fall in urinary ammonia excretion. Considerable individual variation was observed when post-acetazolamide urine ammonia excretion and renal vein ammonia release, obtained at each sampling period, was totaled and compared with resting measurements. However, this variability might be expected since renal vein ammonia release was calculated from instantaneous values obtained from one renal vein, whereas urine was collected from the bladder over ten-minute periods. Nevertheless, the decrease in urine ammonia excretion was approximated by the increase in renal vein ammonia release in most periods, suggesting that total renal ammonia production was not significantly altered.

Although the magnitude of urinary ammonia excretion has been correlated with urine $\mathrm{pH}$ in several species, including man, previous studies utilizing carbonic anhydrase inhibition have been limited to relatively long-term observations. It is apparent from the present investigation that tubular $\mathrm{pH}$ rises within the first ten minutes after intravenous acetazolamide administration and that urinary ammonia excretion falls concomitantly. In contrast, the change in arterial $\mathrm{pH}$ was relatively delayed. Renal venous $\mathrm{pH}$ and $\mathrm{CO}_{2}$ content measured in four patients after acetazolamide showed values which were similar to those of arterial blood. Present evidence suggests that the excretion of ammonia into urine may occur by a process of passive diffusion which, in acute experiments, is conditioned by urine $\mathrm{pH}$ (3-8). Since cells in general are permeable to the molecular species $\mathrm{NH}_{3}$, it would seem reasonable to assume that the ammonia produced within the renal tubular cells diffuses out of the cell with equal facility into tubular urine and into renal venous blood. The relative amounts captured by each should therefore be dependent upon their respective $\mathrm{pH}$ 's and fluid flows. In the present study, acetazolamide rapidly increased urine $\mathrm{pH}$ and decreased ammonia release into the urine; release into the renal vein increased. Additional support for a primary shift in the rate of diffusion of ammonia may be obtained from a consideration of Patients G.J. and J.P. Patient G.J., who exhibited the lowest resting urine $\mathrm{pH}$, showed the greatest reciprocal change in urine and renal vein ammonia after acetazolamide. In contrast, the smallest reciprocal change was noted in Patient J.P., who showed the highest resting urine $\mathrm{pH}$. This suggests that urine $\mathrm{pH}$ is not only an important determinant of urinary ammonia excretion but may also influence the amount of ammonia liberated into the renal vein, at least during the immediate period following intravenous acetazolamide administration.

It is emphasized that the relationship between urine $\mathrm{pH}$ and renal ammonia partition reported in this study need not apply to more chronic situations. Current concepts of renal ammonia production suggest that the magnitude of ammonia release into urine and renal vein over prolonged periods would be influenced by such factors as renal substrate concentration and enzyme activity (33). In addition, it would seem likely that the well recognized alterations in amino acid metabolism (34) exhibited by individuals with liver disease, and their propensity to develop electrolyte imbalance (35) might influence renal ammonia formation in such patients. 


\section{SUMMARY}

The release of ammonia into the right renal vein was measured in 11 patients with liver disease, before and after the intravenous administration of $500 \mathrm{mg}$ of Diamox (acetazolamide). Concomitant measurements of ammonia excretion into urine were obtained in five patients.

The observed increase in mean arterial ammonia concentration after acetazolamide was associated with a prompt twofold increase in the release of ammonia into the renal vein, which was maintained throughout the 60 minute post-acetazolamide period. Renal blood flow remained essentially unchanged throughout the entire study.

The abrupt increase in renal ammonia release was associated with an equally rapid decrease in urine ammonia excretion, which attained maximum significance 20 minutes after acetazolamide and inversely approximated the increase in release into the renal vein. The reduction in urine ammonia excretion could also be correlated with a rise in urine $\mathrm{pH}$.

These observations seem best explained by a shift in the partition of ammonia produced by the kidney between urine and renal vein. In addition, they suggest that acute changes in urine $\mathrm{pH}$ may also influence the amount of ammonia liberated into the renal vein.

\section{ACKNOWLEDGMENT}

The authors wish to thank Dr. Henry Kamin for suggestions and critical review of the manuscript.

\section{REFERENCES}

1. Bessman, S. P. Ammonia metabolism in animals in A Symposium on Inorganic Nitrogen Metabolism, W. D. McElroy and B. Glass, Eds. Baltimore, Johns Hopkins Press, 1956, p. 408.

2. Nash, T. P., Jr., and Benedict, S. R. The ammonia content of the blood, and its bearing on the mechanism of acid neutralization in animal organism. J. biol. Chem. 1921, 48, 463.

3. Briggs, A. P. Functional activity of renal epithelium in certain types of nephritis as indicated by secretion of ammonia. Arch. intern. Med. 1937, 60, 193.

4. Wolf, A. V. Renal regulation of water and some electrolytes in man, with special reference to their relative retention and excretion. Amer. J. Physiol. 1947, 148, 54.

5. Pitts, R. F. Renal excretion of acid. Fed. Proc. 1948, $7,418$.
6. Leonard, E., and Orloff, J. Regulation of ammonia excretion in the rat. Amer. J. Physiol. 1955, 182, 131.

7. Clarke, E., Evans, B. M., MacIntyre, I., and Milne, M. D. Acidosis in experimental electrolyte depletion. Clin. Sci. 1955, 14, 421.

8. Orloff, J., and Berliner, R. W. The mechanism of the excretion of ammonia in the dog. J. clin. Invest. 1956, 35, 223.

9. Poppell, J. W., Cuajunco, F., Jr., Horsley, J. S., III, Randall, H. T., and Roberts, K. E. Renal arteriovenous ammonium difference and total renal ammonium production in normal, acidotic and alkalotic dogs. Clin. Res. 1956, 4, 137.

10. Webster, L. T., Jr., and Davidson, C. S. Production of impending hepatic coma by a carbonic anhydrase inhibitor, Diamox. Proc. Soc. exp. Biol. (N. Y.) 1956, 91, 27.

11. Dawson, A. M., deGroote, J., Rosenthal, W. A., and Sherlock, S. The effects of Diamox in ammonia metabolism in liver disease. Clin. Sci. 1957, 16, 413.

12. Webster, L. T., Jr., and Kleinerman, J. Effect of a carbonic anhydrase inhibitor, acetazoleamide (Diamox $\left.{ }^{\circledR}\right)$ on cerebral "ammonium" metabolism of patients with and without hepatic cirrhosis (abstract). J. clin. Invest. 1957, 36, 934.

13. Brown, R. H., Duda, G. D., Korkes, L., and Handler, $P$. A colorimetric micromethod for the determination of ammonia; the ammonia content of rat tissues and human plasma. Arch. Biochem. 1957, 66, 301.

14. Tyor, M. P., and Wilson, W. P. Peripheral biochemical changes associated with the intravenous administration of ammonium salts in normal subjects J. Lab. clin. Med. 1958, 51, 592.

15. Selkurt, E. C. Measurement of renal blood flow. Meth. med. Res. 1949, 1, 196.

16. Bratton, A. C., and Marshall, E. K., Jr. A new coupling agent for sulfanilamide determination. J. biol. Chem. 1939, 128, 537.

17. Rosenthal, T. B. The effect of temperature on the $\mathrm{pH}$ of blood and plasma in vitro. J. biol. Chem. 1948, 173, 25.

18. Van Slyke, D. D., and Neill, J. M. The determination of gases in blood and other solutions by vacuum extraction and manometric measurements. J. biol. Chem. 1924, 61, 523.

19. Van Slyke, D. D., and Sendroy, J., Jr. Studies of gas and electrolyte equilibria in blood. XV. Line charts for graphic calculations by the HendersonHasselbalch equation and for calculating plasma carbon dioxide from whole blood content. J. biol. Chem. 1928, 79, 781.

20. Hickam, J. B., and Frayser, R. Spectrophotometric determination of blood oxygen. J. biol. Chem. 1949, 180, 457.

21. Bradley, S. E., Ingelfinger, F. J., Bradley, G. P., and Curry, J. J. The estimation of hepatic blood flow in man. J. clin. Invest. 1945, 24, 890. 
22. Cargill, W. H. The measurement of glomerular and tubular plasma flow in the normal and diseased human kidney. J. clin. Invest. 1949, 28, 533.

23. Van Slyke, D. D., Phillips, R. H., Hamilton, P. B., Archibald, R. M., Futcher, P. H., and Hiller, A. Glutamine as source material of urinary ammonia. J. biol. Chem. 1943, 150, 481.

24. Nathans, D., Fahey, J. L., and Ship, A. G. Sites of origin and removal of blood ammonia formed during glycine infusion: Effect of L-arginine. J. Lab. clin. Med. 1958, 51, 124.

25. McDermott, W. V., Jr., Adams, R. D., and Riddell, A. G. Ammonia metabolism in man. Ann. Surg. 1954, 140, 539.

26. White, L. P., Phear, E. A., Summerskill, W. H. J., and Sherlock, S. Ammonium tolerance in liver disease: Observations based on catheterization of the hepatic veins. J. clin. Invest. 1955, 34, 158.

27. Berry, J. N., Flanagan, J. F., Owen, E. E., and Tyor, M. P. The kidney as a source of blood ammonia in resting and hyperventilated cirrhotics. Clin. Res. 1959, 7, 154.

28. Summerskill, W. H. J., Wolfe, S. J., and Davidson, C. S. The metabolism of ammonia and $\alpha$-keto- acids in liver disease and hepatic coma. J. clin. Invest. 1957, 36, 361.

29. Tyor, M. P., and Sieker, H. O. Biochemical, blood gas, and peripheral circulatory alterations in hepatic coma. Amer. J. Med. 1959, 27, 50.

30. Webster, L. T., Jr., and Gabuzda, G. J. Ammonia uptake by the extremities and brain in hepatic coma. J. clin. Invest. 1958, 37, 414.

31. Bessman, S. P., and Bessman, A. N. The cerebral and peripheral uptake of ammonia in liver disease with an hypothesis for the mechanism of hepatic coma. J. clin. Invest. 1955, 34, 622.

32. Owen, E. E., and Tyor, M. P. Unpublished observations.

33. Milne, M. D., Scribner, B. H., and Crawford, M. A. Non-ionic diffusion and the excretion of weak acids and bases. Amer. J. Med. 1958, 24, 709.

34. Wu, C., Bollman, J. L., and Butt, H. R. Changes in free amino acids in the plasma during hepatic coma. J. clin. Invest. 1955, 34, 845.

35. Schwartz, R., Phillips, G. B., Gubuzda, G. J., Jr., and Davidson, C. S. Blood ammonia and electrolytes in hepatic coma. J. Lab. clin. Med. 1953, 42, 499. 